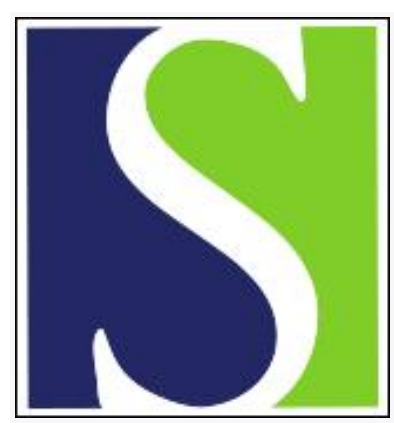

Scand J Work Environ Health 1984;10(6):455-459

https://doi.org/10.5271/sjweh.2298

Issue date: Dec 1984

Completeness of occupational history and occurrences of work-related diseases.

by Koskela RS, Kolari PJ, Jarvinen E, Korhonen $\mathrm{H}$

This article in PubMed: www.ncbi.nlm.nih.gov/pubmed/6535248

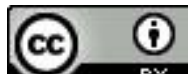




\title{
Completeness of occupational history and occurrences of work-related diseases
}

\author{
by Riitta-Sisko Koskela, MSocSc, Pertti J Kolari, MSc, Erkki Järvinen, MSc, Hannu \\ Korhonen, MSocSc ${ }^{1}$
}

\begin{abstract}
KOSKELA R-S, KOLARI PJ, JÄRVINEN E, KORHONEN H. Completeness of occupational history and occurrences of work-related diseases. Scand J Work Environ Health 10 (1984) 455-459. The lack of complete occupational histories causes underestimates or overestimates of occupational mortality and morbidity. The problem is emphasized in cohorts with high turnover and in cross-sectional study designs. In the study of the effect of selective turnover on occupational mortality and morbidity complete occupational histories were acquired via questionnaire. The sample comprised 1200 current and 1800 alive former workers and the closest relatives of 450 deceased workers and was formed from three exposurelevel cohorts (defined primarily according to the physical demands of the work) of 15714 metal workers hired in 1950-1976. The occupational histories reported in the questionnaires were compared with those collected from employers' personnel records. The coverage of occupational histories was the most accurate for current and former workers with the longest duration of exposure and the least accurate for dead workers and the oldest age groups. The use of complete occupational histories and the combining of the similar levels of exposure through the lifetime produced clear differences in the occurrences of musculoskeletal, respiratory, and cardiovascular diseases between the three levels of exposure. The errors in person-years were smaller than the errors in the duration of exposure; the coverage varied from 51 to $94 \%$. However, misclassification clearly caused cancer cases to be located in classes with too short a period of latency. About $30 \%$ of the deceased workers had reportedly entered the industry before the first follow-up year.
\end{abstract}

Key terms: cardiovascular diseases, health selection, heaviness of work, metal industry, musculoskeletal diseases, respiratory diseases, turnover.

The lack of complete occupational histories causes underestimates or overestimates of occupational mortality and morbidity. The problem is emphasized in cohorts with high turnover and in cross-sectional study designs $(1,2,3,4)$.

In the study of the effect of selective turnover on occupational mortality and morbidity, complete occupational histories were acquired via questionnaire. The sample comprised 1200 current and 1800 alive former workers and the nearest relatives of 450 deceased workers and was formed from three exposure-level cohorts of 15714 metal workers hired in 1950-1976. The cohorts were defined primarily according to the physical demands of the work, heavy (iron foundries), medium (manufacture of metal products), and light (manufacture of electrical devices). The occupational histories reported in the questionnaires were compared with those collected from employers' personnel records. The occurrences of different symptoms and diagnosed diseases were calculated according to both sources of information. A cross-sectional cohort of 1526 workers (who had 1 Department of Epidemiology and Biostatistics, Institute
of Occupational Health, Helsinki, Finland.

Reprint requests to: Ms R-S Koskela, Institute of Occupational Health, Haartmaninkatu 1, SF-00290 Helsinki, Finland. been hired earlier and were still working in 1950) in the three industrial branches was used to determine selection due to disability and mortality.

The coverage of the duration of exposure as registered on employers' records, in comparison with the information reported by the workers, was expressed as percentages of the former to the latter. The coverage was the most accurate for current workers and former workers with the longest duration of exposure and the least accurate for former workers with the shortest duration of exposure (figure 1). Dead former workers had somewhat lower coverage percentages than the corresponding groups of alive workers. The coverage also decreased as the study group aged.

Most of the discrepancies in the duration of exposure were, however, less than two years (68 to $90 \%$ for different groups of current workers and 37 to $85 \%$ for different groups of former workers). The largest discrepancies were found among metal product workers, whose periods of employment were generally longer than those of foundry and electrical workers.

When the occurrences of diagnosed diseases were compared between the three levels of exposure, no great differences were found in the prevalences of diagnosed musculoskeletal, lung, or heart diseases (table 1). The differences were small, especially 
Current workers

Former workers with the longest duration of employment

Former workers with the shortest duration of employment

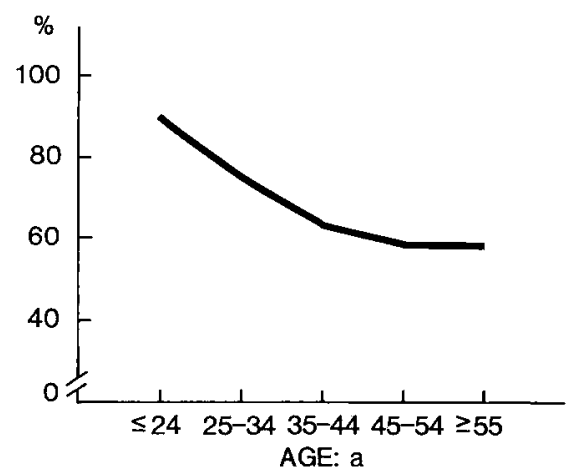

Figure 1. Coverages of exposure times on employers' records as opposed to those notified by employees (percentage of former to latter).

I. Musculoskeletal diseases

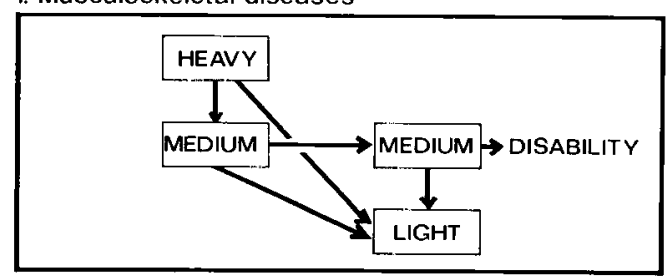

II. Respiratory diseases

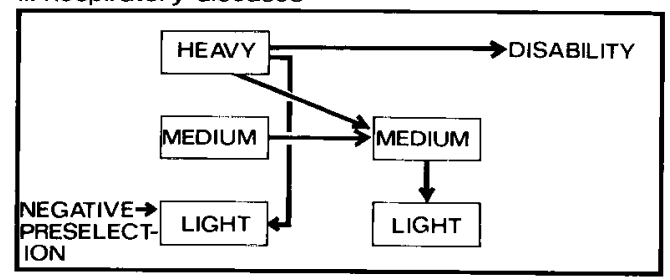

III. Cardiovascular diseases

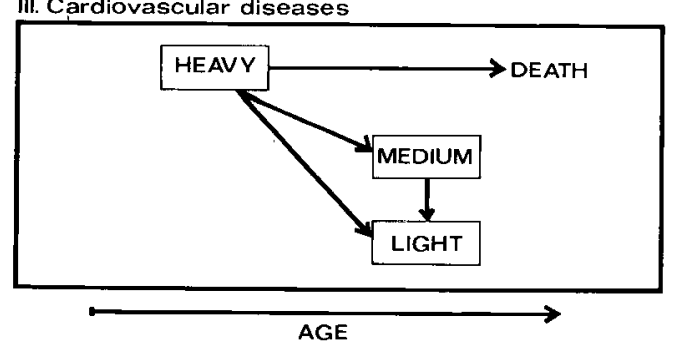

Figure 3. Selection by different diseases between the levels of exposure.
Table 1. Occurrences of diagnosed disease (\%) among current workers at the three levels of exposure.

\begin{tabular}{lccc}
\hline Disease & Foundry & $\begin{array}{c}\text { Metal } \\
\text { products }\end{array}$ & $\begin{array}{c}\text { Electrical } \\
\text { devices }\end{array}$ \\
\hline Any musculo- & & & \\
skeletal disease & 36 & 35 & 16 \\
Any lung disease & 37 & 35 & 28 \\
Any heart disease & 15 & 15 & 8 \\
\hline
\end{tabular}

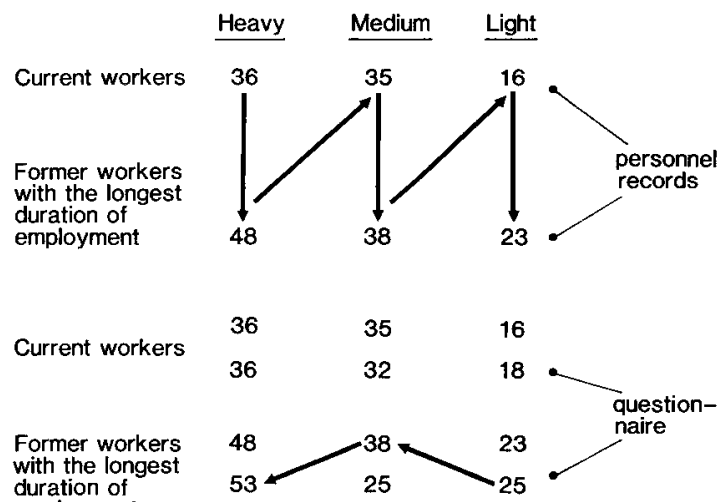

duration of

Figure 2. Occurrences of musculoskeletal diseases (\%) according to the level of exposure.

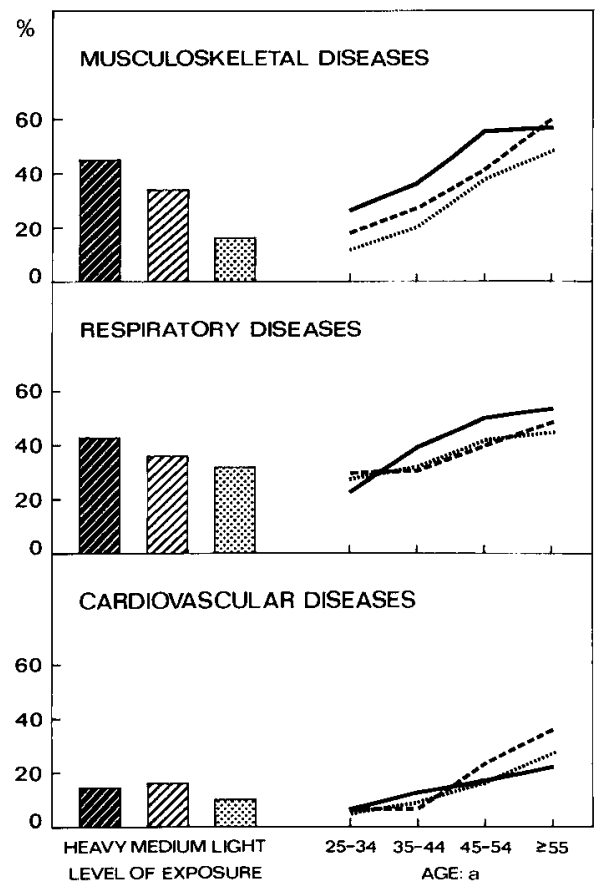

Figure 4. Occurrences of diagnosed diseases (\%) according to the main level of exposure during lifetime. 
between the heavy and the medium levels. But, when the groups of former workers were included, differences in occurrences were revealed. For example, the prevalences of musculoskeletal diseases among current workers were $36 \%$ for the heavy level, $35 \%$ for the medium level, and $16 \%$ for the light level of exposure (figure 2). The corresponding rates among former workers with the longest duration of employment were 48,38 , and $23 \%$. This series of prevalence rates not only shows differences between current and former workers, but also a process of health selection from one level of exposure to another.

However, the presented rates are still overestimates or underestimates, because they were calculated for the original exposure levels defined on the basis of the main occupation registered on employers' records. When the complete occupational histories acquired by questionnaire were used, with the main occupations classified according to the three levels of exposure, the occurrences could be placed on the correct levels, and the transfers due to misclassification became clear.

The selection process was different for different diseases and was manifested as different stages of disease (figure 3 ).

Ache or pain in the musculoskeletal system within the last 12 months was more frequent among foundry and metal product workers than among electrical workers. Both currently employed and former foundry workers had significantly more earlier diagnosed musculoskeletal diseases than electrical workers. Younger age classes of foundry workers (under 45 years) had a higher occurrence of musculoskeletal diseases than the same age classes of metal product workers; in older age classes the opposite was true. The former foundry workers had more diagnosed musculoskeletal diseases than the current workers; there was no such difference among metal product workers. The results indicated that musculoskeletal diseases clearly lead to selection away from heavylevel work. This conclusion was supported also by disability due to musculoskeletal diseases, which showed a greater excess in the cross-sectional cohort than in the longitudinal one.

Both the current and the former foundry workers had higher rates of chronic bronchitis (in different smoking categories) than the corresponding groups of metal product and electrical workers. Selection away from heavy-level exposure was indicated by higher occurrences of chronic bronchitis among former than current foundry workers from the age class of 45-54 years on. A similar trend was found among metal product workers, but the rates were lower. Diagnosed lung diseases were also more frequent at the heavy level than at the medium or light level of exposure. Health selection during employment was also suggested by the disability rates for respiratory diseases. Respiratory diseases were more frequent than expected in both the cross-sectional and the longitudinal cohorts, but the excess was greater in the cross-sectional one. Negative health selection was shown as higher occurrences of diagnosed emphysema and bronchitis in the youngest age class of electrical workers.

Angina pectoris (irrespective of smoking habits) was more prevalent among both current and former foundry workers than among the electrical workers, whereas the difference between the foundry workers and the metal product workers was not significant. Former foundry workers had somewhat higher occurrences than current workers. The same was true for the metal product workers. The current electrical workers had higher occurrences than the former electrical workers. This result may reflect negative health selection to the light level from the heavy and medium levels of exposure. Analysis according to the complete occupational history showed that workers at the heavy level had more diagnosed cardiovascular diseases in the younger age classes and among the workers at the medium level these diseases were more common in the older age classes, a finding which can be interpreted as the result of selection. Disability due to all cardiovascular diseases and the number of cases of coronary heart disease alone were significantly lower for foundry workers than for the active male population. This finding can be explained both by health selection into and out of heavy-level exposure and by mortality rates for the older age groups.

The use of complete occupational histories and the combination of the similar levels of exposure through the lifetime produced clearer differences in the occurrences of musculoskeletal, respiratory, and cardiovascular diseases between the three levels of exposure (figure 4).

The heavy level had significantly higher occurrences of any previously diagnosed musculoskeletal disease which had prevented work for at least one month than the medium $(p<0.01)$ or the light ( $p<0.001$ ) level of exposure, and the medium level had higher occurrences than the light level of exposure $(\mathrm{p}<0.01)$.

The prevalences of all diagnosed lung diseases were the highest at the heavy level $(42 \%)$, the second highest at the medium level $(36 \%)$, and the lowest at the light level of exposure $(32 \%)$. The differences between the branches were not statistically significant.

Previously diagnosed heart diseases were also studied according to the level of exposure. This analysis did not reveal great differences between the three levels. The medium level had the highest prevalence $(16 \%)$, the heavy level the second highest $(14 \%)$, and the light level the lowest $(10 \%)$. However, workers 45 years or older at the medium level of exposure had significantly more previously diagnosed heart diseases than workers of the same age at the light level. 
Current workers
Former workers with
the longest duration of employment

Former workers with the shortest duration of employment

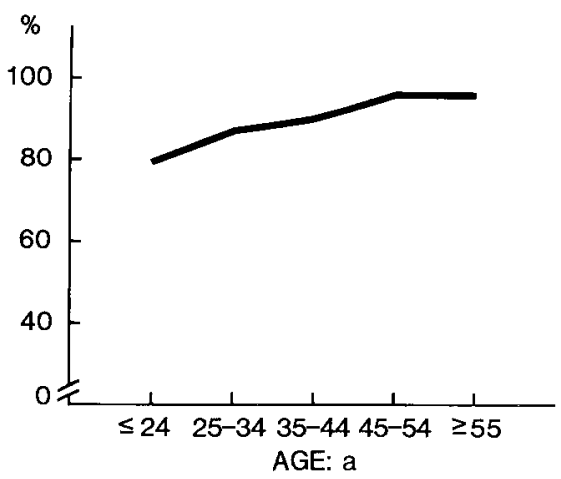

Figure 5. Coverages of person-years on employers' records as opposed to those notified by employees (percentage of former to latter).

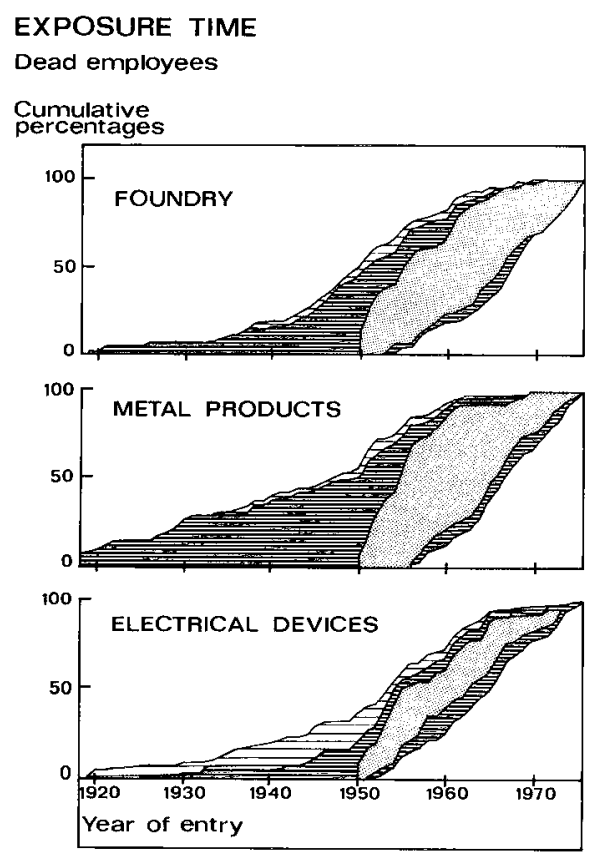

Data from

$\square$ personnel records

inquiry sent to the nearest relatives inquiry exposure in other metal work)

Figure 6. Annual cohort entries of dead employees according to employers' records and inquiries.
Complete occupational histories were available only for the samples. The effect of the lack of complete histories was estimated from calculations of person-years, both from the years of entry registered on the employers' records and those reported until the end of the follow-up. The coverage of personyears (percentage of the former to the latter) varied from 51 to $94 \%$ (figure 5). The errors in personyears were clearly smaller than the errors in the durations of exposure. The greatest loss of person-years was found for the deceased metal product workers $(49 \%)$. On the contrary to the coverage of exposure time, the coverage of person-years increased as the study groups aged.

On the basis of the questionnaire data and regardless of the quality of the response, the proportion of workers who had entered the branch under study before the first year of follow-up (1950) was the highest for deceased workers (about $30 \%$ ), especially for metal product workers (note also confounding exposure in the metal industry for the electrical workers) (figure 6). Among alive workers the proportion of workers who had entered before 1950 was the highest for former workers with the longest duration of exposure (figure 7). This finding emphasizes the importance of complete occupational histories when mortality results are calculated according to a period of

\section{EXPOSURE TIME}

Alive employees (metal products)

Cumulative
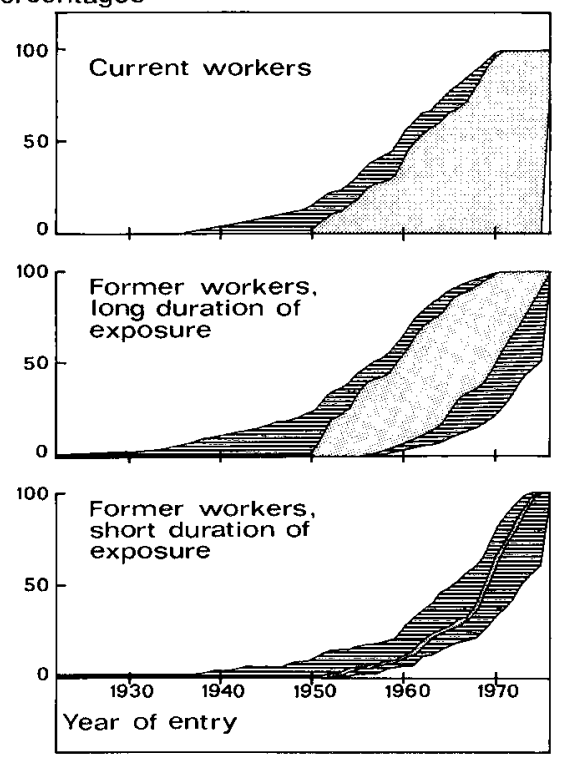

Data from

$D$ personnel records

汪quiry

Figure 7. Annual cohort entries of alive employees according to employers' records and inquiries. 
latency. For instance, misclassification can cause cancer cases to be located in classes with too short a period of latency or can even entirely exclude them from the study group (if criteria are set for the minimum period of exposure and/or latency). This was the case in the cohort of metal product workers, where the only excessive mortality from tumors was found during the first two five-year periods of follow-up. At least $50 \%$ of the deceased metal product workers who had died from tumors had entered the industry before the first year of follow-up.

\section{References}

1. Fox AJ, Collier PF. Low mortality rates in industrial cohort studies due to selection for work and survival in the industry. Br J Prev Soc Med 3 (1976) 225-230.

2. Gamble J, Spirtas R. Job classification and utilization of complete work histories in occupational epidemiology. J Occup Med 18 (1976) 399-404.

3. Koskela R-S, Luoma K, Hernberg S. Turnover and health selection among foundry workers. Scand J Work Environ Health 2 (1976): suppl 1, 90-105.

4. Tola S, Koskela R-S, Hernberg S, Järvinen E. Lung cancer mortality among iron foundry workers. J Occup Med 21 (1979) 753-760. 